\title{
Provider-Patient Interactions as Predictors of Lifestyle Behav- iors Related to the Prevention and Management of Diabetes
}

\author{
Brenda Robles ${ }^{1 *}$, and Tony $\mathrm{Kuo}^{2-4,}$
}

1 Division of Chronic Disease and Injury Prevention, Los Angeles County Department of Public Health, 3530 Wilshire Blvd, $8^{\text {th }}$ Floor, Los Angeles, CA 90010, USA

2 Department of Family Medicine, David Geffen School of Medicine at UCLA, 10880 Wilshire Blvd, Suite 1800, Los Angeles, CA 90024, USA; tkuo@mednet.ucla.edu

3 Department of Epidemiology, UCLA Fielding School of Public Health, P.O. Box 951722, Los Angeles, CA 90095, USA

4 Population Health Program, UCLA Clinical and Translational Science Institute, 10833 Le Conte Ave, BE-144 CHS, Los Angeles, CA 90095, USA

* Correspondence: brrobles@ph.lacounty.gov

\begin{abstract}
Growing evidence suggests that healthcare provider advice may increase tobacco cessation, reduce alcohol use, and improve the adoption of desirable lifestyle behaviors among patients. However, how brief interventions and other provider-patient interactions can shape cumulative adoption of multiple modifiable behaviors is less well studied for diabetes prevention and control. Using weighted internet panel survey data from a large socio-demographically diverse urban population in the United States $(n=1,003)$, the present study describes differences in group characteristics among those who had been "ever diagnosed" with prediabetes/diabetes versus those who had not. It also examines the associations between the cumulative adoption of lifestyle behaviors and each of the following: a) lifetime prediabetes/diabetes diagnosis; b) brief lifestyle intervention exposure (i.e., received provider advice/encouragement); and c) recent provider-patient communication about diabetes. There were several group differences in "ever diagnosed" prediabetes/diabetes status by age, employment status, health status, nutrition knowledge, blood pressure/hypertension diagnosis, and diabetes-related health behaviors $(p<0.05)$. Each of the three provider-patient interactions of interest were positively associated with a higher cumulative sum of adopted modifiable lifestyle behaviors for diabetes prevention and management. Results suggest that provider advice/provider-patient interactions of any type can have a salutary impact on whether individuals with prediabetes or type 2 diabetes will engage in recommended lifestyle behavior modifications.
\end{abstract}

Keywords: type 2 diabetes; lifestyle behaviors; healthcare provider advice; provider-patient interactions; health care setting; brief lifestyle intervention exposure; diabetes prevention; diabetes management.

\section{Introduction}

Despite decades of strong empirical evidence linking lifestyle choices such as diet, physical activity, smoking, and drinking to the risk of developing type 2 diabetes mellitus (T2DM) or its control [1], getting individuals to adhere to recommended lifestyle behaviors remains a major public health challenge. This is especially true in the United States (U.S.), where adherence to national recommendations on lifestyle actions to prevent and control cardiovascular and other chronic diseases has been historically low [2]. While some progress has been made in getting Americans to engage in healthier lifestyle behaviors in the last decade [3], sociodemographic disparities persist. This is an issue recently highlighted in the American Heart Association's 2030 Impact Goal [4].

With the global prevalence of T2DM forecasted to reach 7,862 individuals per 100,000 by the year 2040 [5], it has become a global priority to disseminate cost-effective and easier-to-implement interventions that can close the gap on these existing T2DM-related 
disparities. Provider-patient interactions in the health care setting are such an effort and represent a promising avenue to improving population-level lifestyle behaviors, especially for groups that have low levels of adherence to recommended lifestyle behaviors. This is no more apparent than the large body of research demonstrating advice from a physician or other health professionals in the health care setting (e.g., nurses, dieticians, occupational therapists, etc.) can lead to better lifestyle behavioral outcomes among patients. For example, brief advice from a physician or a healthcare provider with similar credibility has been shown to be effective in reducing alcohol use among patients exposed to this brief intervention [6,7]. Likewise, emerging research points to how provider advice can improve diet and/or physical activity [8,9], as well as other related health outcomes such as for overweight/obesity [10]. Other forms of provider-patient interactions that appear to be promising in getting individuals to improve their lifestyle choices include brief lifestyle interventions, whereby physicians/other healthcare providers offer more thorough counseling and patient aids (e.g., assessment and direct feedback, distribution of educational materials) [11, 12]. However, the context and impact of these brief lifestyle interventions for diabetes prevention and management are less well understood than for brief interventions on tobacco cessation and problem drinking; they may also be more complex and less straightforward to implement in practice $[13,14]$. Furthermore, the linkages between different types of provider-patient interactions and T2DM-related lifestyle behaviors remain unclear, especially among sociodemographically diverse populations.

The present study sought to address these gaps in health services knowledge and public health practice by using cross-sectional data collected from an internet panel survey conducted in one of the largest sociodemographically diverse jurisdictions in the U.S. To the best of our knowledge, this study appears to be the first to describe and examine the associations between three types of provider-patient interactions (i.e., lifetime prediabetes/diabetes diagnosis, brief lifestyle intervention exposure, and recent provider-patient communication about diabetes) and cumulative adoption of lifestyle behaviors for the prevention or management of diabetes. We hypothesized that any type of provider-patient interaction will lead to the adoption of several of these lifestyle behaviors, even after controlling for sociodemographic and other individual health characteristics.

\section{Materials and Methods}

\subsection{Dataset}

The internet panel survey dataset used in the present study is a cross-sectional database that has been described previously in another project [15]. The Los Angeles County Department of Public Health (DPH) commissioned a survey firm to assess the sodiumrelated knowledge, attitudes, and health behaviors of Los Angeles County residents who were a member of a panel of online subscribers during August 4-12, 2016. While the initial objective of the survey was to inform local sodium reduction efforts, relevant information about diabetes and other health conditions was also collected. Respondents from the panel were enrolled via the survey firm's sampling partner, an organization that worked with a diverse group of globally recognized and business-focused brands to invite everyday consumers into the survey. Only individuals who had a pre-existing relationship with the sampling partner were recruited and enrolled in the study. This recruitment strategy was designed to ensure the highest level of panel quality and representativeness, and to guard against duplication, involvement by fraudulent respondents, and disruptions from professional survey takers.

Only panel subscribers who were 18 years of age or older and who were a resident of Los Angeles County at the time of the survey were emailed an invitation to participate. Interested subscribers were asked to complete a series of screening sociodemographic questions to further determine their eligibility for the survey. This sociodemographic information was part of a targeted quota sampling strategy that enrolled respondents into each of the desired categories (i.e., age, sex, race/ethnicity, income, and Service Planning 
Area) until saturation was reached. The targets were based on data from the 2013 American Community Survey [16] and the 2011 Los Angeles County Health Survey [17]. The quota targets were also used in the survey weighting process which considered the age, income, sex/ethnicity, race, children in the household, poverty-level demographic distributions, and Service Planning Area of the recruited residents. Although the 2013 American Community Survey was the mainstay of the survey firm's quota and weighting schemes, the 2011 Los Angeles County Health Survey provided additional information on demographic trends/distributions that were not available or entirely complete in the census (i.e., Service Planning Area-level income, marital status, household size, and number of children in the household).

The survey firm piloted and fielded the survey instrument before disseminating it to the respondents. In total, 2,837 panel subscribers clicked on the invitation link, of which 1,003 completed the survey, achieving an adjusted participation rate of $\sim 35 \%$. This participation rate was comparable to many web- and population-based surveys that utilized similar sampling approaches $[17,18]$. This study was reviewed and approved by the Los Angeles County Department of Public Health Institutional Review Board.

\subsection{Measures}

\subsubsection{Dependent variable}

The primary outcome variable was the cumulative sum of adopted modifiable lifestyle behaviors that are thought to be effective or can have a salutary impact on preventing and/or managing diabetes [19-21]; in this study's analyses, 'diabetes' refers to T2DM. This dependent variable was measured by asking respondents to indicate if they were "doing any of the following to help prevent or control diabetes: 1) exercising regularly; 2) controlling their weight; 3) trying to lose weight; 4) reducing their alcohol consumption; 5) reducing their carbohydrate intake; 6) reducing their sugar intake; and/or 7) reducing the number of cigarettes they smoke." For each lifestyle behavior, respondents had an option to indicate "yes" by clicking on a check box placed next to the behavior (coded as 1); unchecked boxes were classified as "no" and coded as 0 . Responses to the seven behaviors were summed and analyzed as a count variable in the multivariable (negative binomial) regression analyses. Variable scores ranged from 0 to 7 , with a higher score indicating a respondent engaging in a greater number of adopted modifiable lifestyle behaviors (cumulative sum) that are protective against diabetes/diabetes complications.

\subsubsection{Independent variables}

Three distinct provider-patient interaction variables were examined as hypothesized predictors (i.e., primary regressors in each of the respective negative binomial models) of the aforementioned cumulative sum of adopted modifiable lifestyle behaviors:

Lifetime prediabetes/diabetes diagnosis (status). This variable was measured by asking respondents the following two questions: 1) "Have you ever been told by a doctor or other health professional that you have prediabetes?"; and 2) "Have you ever been told by a doctor or other health professional that you have diabetes?" For each question, respondents had the option to indicate "yes" (coded as 1) or "no" (coded as 0 ). Responses for each of the two questions were summed into a variable with scores ranging from 0 to 3 . A score of 0 indicated that a respondent had not been ever diagnosed with prediabetes or diabetes; a score of 1 indicated that a respondent had been ever diagnosed with either prediabetes or diabetes; and a score of 3 indicated that a respondent had been ever diagnosed with both prediabetes and diabetes. Given few respondents indicated they had been ever diagnosed with both conditions ( $\mathrm{n}=10)$, the variable was dichotomized as: 'had not been ever diagnosed' (reference) for a score of 0 and 'ever diagnosed' for scores of 2 or 3 . 
Brief lifestyle intervention exposure. This variable assessed the number of modifiable lifestyle behaviors that a respondent was encouraged to engage in by a doctor or other health professional - i.e., behaviors thought to be effective or can have a salutary impact on preventing and/or managing T2DM. Respondents were asked, "And, which of the following has your doctor or another health professional ever encouraged you to do: 1) increase physical activity; 2) lose weight; 3 ) reduce sodium or salt intake; 4) reduce sugar intake; and 5) reduce carbohydrate intake." For each lifestyle behavior, respondents had an option to indicate "yes" by clicking on a check box placed next to the behavior (coded as 1); unchecked boxes were classified as "no" and coded as 0 . These responses were then summed and analyzed as a count variable in the negative binomial regression analyses. Scores ranged from 0 to 5 , with a higher score indicating a respondent having greater exposure to brief lifestyle interventions.

Recent diabetes provider-patient communication(s). This variable captured whether a respondent had spoken with a healthcare provider about prediabetes/diabetes at least once in the past year. Respondents were asked the following two questions: 1) "In the past 12 months, how many times, if ever, have you [spoken] to a doctor or other health professionals, such as a nurse or pharmacist, about prediabetes?"; and 2) "In the past 12 months, how many times, if ever, have you [spoken] to a doctor or other health professional, such as a nurse or pharmacist, about diabetes?" Response options for each question were "1-2 times," "3-5 times," "5 or more times," and "have not in the past 12 months." These responses were then dichotomized as 'no, have not spoken to a health care provider in the past year' (coded as 0 [reference]) if they responded, "have not in the past 12 months" to both questions; all other responses were categorized as 'yes, have spoken to a health care provider in the past year' (coded as 1).

\subsubsection{Covariates}

The multivariable (negative binomial) regression analyses controlled for the following respondent characteristics:

Health status. The following measures of health status were included in the negative binomial regression analyses: 1) high blood pressure/hypertension status - ever diagnosed with high blood pressure/hypertension by a doctor or health care provider [reference] versus not ever diagnosed; 2) self-reported health status - good/fair/poor [reference] versus excellent/very good; 3) self-reported weight management status - trying to lose weight [reference] versus not trying to lose weight; and 4) self-reported physical activity level - not very active [reference] versus active. The question used to assess high blood pressure/hypertension aligns with an item used in a prior study conducted in Los Angeles County [22]. Similarly, prior studies have also assessed self-reported health status [13, 20] and self-reported physical activity levels [23] among Los Angeles County residents using questions similar to those used in the present study. Finally, the question used to assess self-reported weight management status, "Are you currently [trying to lose weight/control weight] to help prevent or control high blood pressure" was internally developed; it was pretested prior to field implementation.

Nutrition knowledge. This was assessed by asking respondents three questions related to calorie and sodium knowledge. The first two questions asked respondents to indicate their knowledge of daily recommended calories limits (i.e., "In general, an average adult should consume no more than [fill in] calories per day") and knowledge of daily recommended milligrams of sodium limits (i.e., "In general, an average adult should consume no more than [fill in response] milligrams of sodium per day?"). Responses for both questions were reported as whole number responses. For calorie knowledge, responses of 1800 to 2400 calories were classified as 'accurate knowledge' and coded as 1, while all other responses outside of this range were classified as 'inaccurate knowledge' and coded as 0 . 
The same question and approach used in the present study to dichotomize the calorie knowledge variable has been described elsewhere previously [15]. Similarly, for sodium knowledge, responses of 1500 to 2300 milligrams of sodium were classified as 'accurate knowledge' (coded as 1) and all other responses as 'inaccurate knowledge' (coded as 0 ). The same question and approach to the sodium knowledge variable have also been previously described $[15,22]$. A third question showed respondents three distinct nutrition fact labels (i.e., with different serving sizes, servings per container, sodium values, ) corresponding to three sauces (i.e., Sauce A, Sauce B, and Sauce C). Respondents were asked to indicate which of the three sauces had the least amount of sodium per cup. Responses were coded as 1 if the respondent chose Sauce B (i.e., correct answer because it was the nutrition label with the lowest sodium content) and coded as 0 if the respondent chose Sauce A or Sauce C (i.e., incorrect answer because these nutrition labels contained a higher sodium content than Sauce B). This same question and categorization approach was used in a previous study of Los Angeles County residents [15]. Since the responses to the three questions were highly correlated, they were summed into a variable with scores ranging from 0 to 3, which were categorized as follows: 'low knowledge' (reference) for a score of 0 (i.e., the respondent answered zero of three questions correctly), 'medium knowledge' for a score of 1 (i.e., the respondent answered one of three questions correctly), 'high' for a score of 2 (i.e., respondent answered two of three questions correctly), and 'very high' for a score of 3 (i.e., the respondent answered three of three questions correctly).

Frequency of eating restaurant meals. Using questions adapted from a previous study [24], respondents were asked to indicate the number of times in a typical week they eat a meal from: 1) "a sit-down restaurant such as: neighborhood restaurants, chains and fine dining establishments including take-out and delivery"; and b) "a meal from a fast-food restaurant or chain such as: McDonald's, Taco Bell, Subway, or similar including take-out and delivery." Responses to each of the two questions were reported as a whole number value and summed. In the negative binomial regression analyses, these responses were analyzed as a count variable. Scores ranged from 0 to 30, with a higher score indicating greater weekly fast-food/sit-down restaurant meal consumption.

Food ordering/selection considerations. Three distinct variables capturing respondents' food ordering/selection decision-making behaviors were included in the negative binomial regression models. For the first variable, respondents were asked to indicate how important each of the following are to them when deciding what food to order at a restaurant: 1) amount of salt/sodium in a meal; 2) amount of carbohydrates in a meal; 3) amount of fat in a meal; 4) amount of sugar in a meal; and 5) amount (number) of calories in a meal. Responses for each nutrient were reported as a 5-point Likert scale ranging from "extremely important" (coded as 5) to "not at all important" (coded as 1). Then the scores for each of the five nutrient questions were summed. Scores ranged from 5 to 25, with a higher score indicating a greater level of importance. For the second variable, respondents were asked, "When buying packaged products, how often do you use a food label or Nutrition Facts label to help you decide what food to purchase?" Response options were reported as a 5-point Likert scale ranging from "always" to "never." Responses were dichotomized as "never to about half the time' (reference) if a respondent indicated "never," "less than half of the time," or "about half of the time" and 'always/most of the time' if a respondent indicated "always" or "most of the time." For the third variable, respondents were asked if they were "currently watching or reducing [their] salt intake." Responses options included "no" (reference) and "yes."

Sociodemographic characteristics. The following characteristics were included in the analyses: sex (male [reference], female), age (18-44 years [reference], 45-64 years, 65 years or older), race/ethnicity (White [reference], Black, Hispanic/Latino, Asian/Native Hawaiian or Pacific Islander [ANHOP]), education status (obtained professional degree 
[reference], high school or less/vocational school, some college or graduated from 2-year college with a degree, graduated with a degree from 4-year college), employment status (full-time [reference], part-time, unemployed/student/homemaker, retired), and marital status (not married or in a domestic partnership [reference], married or in a domestic partnership).

\subsection{Data Management and Analyses}

STATA version 14.1 (StataCorp LP, College Station, Texas) was employed to clean, manage, and analyze the internet panel survey dataset; the resulting analyses included the use of survey weights. A conceptual framework informed by a literature review guided the study analyses (framework not shown). Descriptive, correlation, factor, and multicollinearity analyses were carried out to inform variable selection and operationalization. This included examining the univariate distributions of all variables included in the analyses (i.e., histograms, frequencies, median, range, standard deviation). A final analytic sample was generated by removing sparse data, outliers, and missing responses for any of the final variables of interest; due to small cell sizes, respondents who identified themselves as "American Indian/Alaskan Native" or "other" were dropped. In the multivariable regression analyses, income was dropped due to it being highly correlated with education. All descriptive and regression analyses were carried out using the final analytic sample ( $\mathrm{n}=953)$.

Descriptive analyses were performed to examine weighted percentages and frequencies for categorical variables and weighted medians and interquartile range for continuous/count variables in: a) the full sample; and b) by whether a respondent had been ever diagnosed with prediabetes/diabetes by a doctor or health professional. To compare sociodemographics, health status, nutrition knowledge, and food consumption/selection/ordering behaviors among respondents who had been ever diagnosed with prediabetes/diabetes versus those who had not, weighted Pearson chi-squared and Kruskal-Wallis tests were performed for categorical and continuous/count variables, respectively. P-values $<0.05$ were considered statistically significant in these analyses.

Multivariable regression models were constructed to examine the associations between the dependent variable (cumulative sum of adopted modifiable lifestyle behaviors) and the three independent variables of interest (i.e., lifetime prediabetes/diabetes diagnosis, brief lifestyle intervention exposure, and recent provider-patient communication about diabetes). To generate incidence rate ratios (IRRs) and 95\% confidence intervals (CIs), both simple and full negative binomial regression models were generated, excluding and including covariates, respectively. The negative binomial regression analysis was the model of choice because the dependent variable (outcome) was an over-dispersed count.

\section{Results}

\subsection{Descriptive Results}

Survey respondent sociodemographic, health status, nutrition knowledge, and provider-patient communication characteristics are presented in Table 1. In the full sample, over half of respondents were female $(51.4 \%)$, between $18-44$ years of age $(53.8 \%)$, and currently married/in a domestic partnership (54.5\%). The majority were also Hispanic/Latino $(38.5 \%)$, employed full-time $(48.4 \%)$, had a high school or vocational level of education $(35.6 \%)$, and had an annual income $<\$ 50,000(45.3 \%)$. Most respondents reported having good/fair/poor health status (58.1\%), trying to lose weight $(55.6 \%)$, and were physically inactive $(80.4 \%)$. The majority of respondents had 'low' nutrition knowledge related to calories and sodium (41.3\%). About $37.5 \%$ of respondents correctly identified daily 
calorie recommendations for adults (data not shown in the table), while their ability to accurately identify daily recommended sodium limits in milligrams per day and low sodium foods using a Nutrition Facts label were about $7.8 \%$ and $37.5 \%$, respectively (data not shown in the table). Only about $21.7 \%$ had been ever diagnosed with high blood pressure/hypertension. Overall, there were statistically significant differences by age, employment status, perceived general health status, nutrition knowledge, and high blood pressure/hypertension diagnosis among respondents who had been ever diagnosed with prediabetes/diabetes versus those who had not $(\mathrm{p}<0.05)$.

Table 1. Respondent characteristics from the 2016 internet panel survey in Los Angeles County, by "ever diagnosed" with prediabetes and/or diabetes status

Characteristics

Total

Sociodemographics

Sex

Female

Male

Age

Race

18-44

45-64

65 years or older

White

Black

Hispanic/Latino

ANHOPI

Education

High school or less/vocational

Some college/attended 2-year college

Graduate of 4-year college

Professional degree

Income

$\$ 24,999$ or less

$\$ 25,000-\$ 49,999$

$\$ 50,000-\$ 74,999$

$\$ 75,000-\$ 99,999$

$\$ 100,000$ or more

Employment status

Employed full-time

Employed part-time

Unemployed/student/homemaker

Retired

Marital status

Currently married/domestic partnership

Not currently married or in domestic partnership

\section{Health Status}

Perceived general health status Excellent/very good Good/fair/poor

Self-reported weight management status Not trying to lose weight

Trying to lose weight

Perceived physical activity level Active
Full Sample

'Ever diagnosed'
by a doctor or
health profes-
sional with predia-
betes/

betes/

Weighted \% (n)

$51.4(511)$

48.6 (442)

$53.8(522)$

31.1 (308)

15.2 (123)

33.9 (365)

$11.1(105)$

$38.5(331)$

$16.6(152)$

$35.6(135)$

33.9 (267)

$18.5(349)$

12.0 (202)

22.3 (159)

23.0 (208)

$17.3(172)$

$11.9(125)$

25.6 (260)

48.4 (516)

14.5 (128)

$22.0(173)$

$15.0(136)$

54.4 (495)

45.6 (458)

13.9 (128)

$48.8(65)$

$51.2(63)$

$29.2(48)$

$36.1(46)$

$34.7(34)$

34.5 (43)

14.1 (18)

$38.6(48)$

12.9 (19)

$34.2(21)$

43.4 (47)

14.3 (41)

8.2 (19)

22.4 (22)

32.9 (36)

15.1 (19)

14.3 (21)

15.2 (27)

34.2 (52)

18.0 (20)

13.8 (19)

34.0 (37)

56.1 (71)

43.9 (57)

'Had not been ever
diagnosed' by a
doctor or health
professional with
prediabetes/

\begin{tabular}{cc}
\hline Weighted \% $(\mathrm{n})$ & Weighted $\%(\mathrm{n})$ \\
\hline $\mathbf{1 3 . 9 ( 1 2 8 )}$ & $\mathbf{8 6 . 2 ( 8 2 5 )}$
\end{tabular}

0.6347

$51.8(446)$

$48.2(379)$

$57.7(474)$

$30.3(262)$

$12.0(29)$

$33.8(322)$

$10.6(87)$

$38.5(283)$

17.1 (133)

35.8 (114)

$32.4(220)$

19.2 (308)

12.6 (183)

22.3 (137)

21.4 (172)

$17.6(153)$

11.5 (104)

27.3 (233)

50.7 (464)

$14.0(108)$

23.3 (154)

12.0 (99)

54.1 (424)

45.9 (401)

24.3 (37)

75.7 (91)

44.8 (418)

55.3 (407)

$38.6(45)$

61.4 (83)

45.3 (376)

54.7 (449)

17.2 (23)
0.7528

$<0.0001$

0.6873

0.1436

0.0844

$<0.0001$

0.0005

0.2978

0.5612
20.0 (207) 
Not very active

Nutrition Knowledge

Calorie and sodium knowledge

Low knowledge

Medium knowledge

High knowledge

Very high knowledge

Provider-Patient Communication

Ever diagnosed by a doctor or health care pro-

vider with high blood pressure or hypertension

No, had not been ever diagnosed

Yes, had been ever diagnosed
$80.4(723)$

$41.3(328)$

$37.5(384)$

$18.3(202)$

$3.0(39)$
$82.8(105)$

$28.4(37)$

$53.0(63)$

$16.1(23)$

$3.0(5)$

$80.1(618)$

$43.4(291)$

$35.0(321)$

$18.6(179)$

$3.0(34)$

0.0100

$<0.0001$

$\begin{array}{lll}78.3(746) & 56.8(68) & 81.8(678) \\ 21.7(207) & 43.2(60) & 18.3(147)\end{array}$

Note: Percentages may not add up to the total or $100 \%$ due to rounding; reported n's and percentages are unweighted.

${ }^{a}$ Chi-squared test.

Other respondent characteristics are presented in Table 2. In the full sample, respondents, on average, reported consuming about 3 fast-food/sit-down restaurant meals per week and generated a median score of 16 (out of 25) for the level of importance they placed on considering the nutritional content of foods when they order food at a restaurant. Use of nutritional labels or nutrition facts labeling when buying packaged foods was low; less than half of them reported using the labels $(40.8 \%)$. A higher percentage indicated currently watching or reducing their salt intake $(56.5 \%)$. There were several findings for the three provider-patient interactions (i.e., independent variables included in the negative binomial regression models). For lifetime prediabetes/diabetes diagnosis (status), less than one-sixth indicated they had been 'ever diagnosed' with prediabetes/diabetes $(13.9 \%)$. For brief lifestyle intervention exposure, on average, a doctor or other health professional had recommended that respondents improve about 1 lifestyle behavior. And for recent diabetes provider-patient communication, only about one-third (36.1\%) had spoken to a doctor or health professional about prediabetes/diabetes within the last year. For the dependent variable of interest (i.e., the cumulative sum of adopted modifiable lifestyle behaviors), on average, respondents reported engaging in 1 modifiable lifestyle behavior to prevent or control diabetes. Except for using a food or nutrition facts label when buying packaged food products, there were significant group differences for all of these characteristics depending on whether or not a respondent had been ever diagnosed with prediabetes or diabetes $(\mathrm{p}<0.05)$.

Table 2. Other respondent characteristics, including self-reported health behaviors, from a 2016 internet panel survey in Los Angeles County, by "ever diagnosed" with prediabetes and/or diabetes status

Full Sample

\section{Characteristics}

\section{Total}

Restaurant food consumption frequency

Number of fast-food and/or sit-down restaurant meals eaten per week

Food ordering/selection considerations When ordering food at a restaurant, level of importance of its nutritional content Frequency of using food label or nutrition facts label when buying packaged products

\begin{tabular}{|c|c|c|}
\hline $\begin{array}{c}\text { 'Ever diagnosed' } \\
\text { by a doctor or } \\
\text { health profes- } \\
\text { sional with pre- } \\
\text { diabetes/ } \\
\text { diabetes }\end{array}$ & $\begin{array}{l}\text { 'Had not ever di- } \\
\text { agnosed' by a } \\
\text { doctor or health } \\
\text { professional with } \\
\text { pre-diabetes/ } \\
\text { diabetes }\end{array}$ & P-Value \\
\hline $\begin{array}{c}\text { Weighted \% (n) } \\
\text { or unweighted } \\
\text { median [IQR] }\end{array}$ & $\begin{array}{c}\text { Weighted \% (n) } \\
\text { or unweighted } \\
\text { median [IQR] }\end{array}$ & \\
\hline $13.9(128)$ & $86.2(825)$ & \\
\hline
\end{tabular}

0.0040

\begin{tabular}{|c|c|c|c|}
\hline & diabetes & etes & \\
\hline $\begin{array}{l}\text { Weighted \% (n) } \\
\text { or unweighted } \\
\text { median [IQR] }\end{array}$ & $\begin{array}{c}\text { Weighted \% (n) } \\
\text { or unweighted } \\
\text { median [IQR] }\end{array}$ & $\begin{array}{c}\text { Weighted \% (n) } \\
\text { or unweighted } \\
\text { median [IQR] }\end{array}$ & \\
\hline $100(953)$ & $13.9(128)$ & $86.2(825)$ & \\
\hline 3 [2] & $3[3]$ & $3[2]$ & 0.0040 \\
\hline
\end{tabular}

$16[5] \quad 17[5] \quad 16[5]$ 
Never to about half the time

Always/most of the time

Currently watching or reducing salt intake

No, not currently watching or reducing

Yes, currently watching or reducing

Brief lifestyle intervention exposure

Number of lifestyle behaviors that a doctor or

health care provider recommended to improve on

\section{Recent diabetes provider-patient communication} Spoke with a doctor or health care provider about pre-diabetes/diabetes at least once in the last year No, have not in the past year Yes, have in the past year

Lifestyle behaviors (cumulative sum adopted) Number of lifestyle behaviors that the respondent is engaging in to prevent or control diabetes

$\begin{array}{lll}59.2(503) & 50.9(62) & 60.5(441) \\ 40.8(450) & 49.1(66) & 39.5(384) \\ & & \\ 43.5(427) & 28.8(36) & 45.9(391) \\ 56.5(526) & 71.2(92) & 54.1(434)\end{array}$

0.0056

$<0.0001$

$1[2] \quad 3[2] \quad 1[2]$

$<0.0001$

$63.9(655)$

$36.1(298)$
$15.6(21)$

$84.4(107)$
$71.7(634)$

$28.3(191)$

Note: Percentages may not add up to the total or $100 \%$ due to rounding; reported n's, medians, and interquartile ranges [IQRs] are unweighted.

${ }^{a}$ Chi-squared test or Kruskall-Wallace test.

\subsection{Results from the Multivariable Regression Analyses}

Table 3 presents results of the negative binomial regression models which examined the associations between the cumulative sum of adopted modifiable lifestyle behaviors and lifetime prediabetes/diabetes diagnosis. In both the simple and full models, respondents who had been 'ever diagnosed' with prediabetes/diabetes reported adopting more modifiable lifestyle behaviors than those who 'had not been ever diagnosed' (simple model Incidence Rate Ratio [IRR] $=1.73$, 95\% Confidence Interval [CI] $=1.49-2.01$ ); full model IRR=1.42, 95\% CI=1.22-1.65). Similar results were observed for the other two hypothesized predictors (i.e., primary regressors in their respective models), brief lifestyle intervention exposure (Table 4) and recent diabetes provider-patient communication (Table 5). There was a positive association between the number of lifestyle behaviors that a doctor or health professional recommended to a respondent and the cumulative sum of adopted modifiable lifestyle behaviors that a respondent reported adopting, in both the simple model (IRR=1.28, 95\% CI=1.22-1.34) and full model (IRR=1.21, 95\% CI=1.15-1.27). And in both the simple and full models, respondents who indicated having recently spoken to a doctor or other health professional within the last 12 months/last year reported adopting more modifiable lifestyle behaviors than their counterparts who had not had a recent communication with a provider regarding diabetes (simple model IRR $=1.84,95 \%$ $\mathrm{CI}=1.58-2.14$ ); full model IRR=1.53, 95\% CI=1.31-1.79). While all three provider-patient interactions were significantly associated with higher cumulative sum of adopted modifiable lifestyle behaviors, it appeared that the magnitude of the effect was highest for those with a 'recent diabetes patient-provider communication,' followed by those with a 'lifetime pre-diabetes/diabetes diagnosis' and then those with a 'brief lifestyle intervention exposure.'

Table 3. Association between the cumulative sum of adopted modifiable lifestyle behaviors to prevent/manage diabetes and lifetime prediabetes/diabetes diagnosis (status): Results from a 2016 internet panel survey of Los Angeles County residents $(\mathrm{n}=953)$

Characteristics

Simple Model

$\operatorname{IRR}(95 \% C I)^{a}$

Full Model

\section{Dependent Variable}

IRR $(95 \% \text { CI })^{a}$

Cumulative sum of adopted modifiable lifestyle behaviors to pre-

vent/manage diabetes

Primary Regressor 
Ever diagnosed by a doctor or health professional with prediabetes and/or diabetes ( $r e f=$ no, had not been ever diagnosed)

Yes, had been ever diagnosed with prediabetes and/or diabetes

\section{Covariates}

Sex (ref=male)

Female

Age (ref $=18-44$ years)

45-64 years

65 years or older

Race/ethnicity (ref=White)

Black

Hispanic/Latino

ANHOPI

Education status (ref=obtained professional degree)

High school or less/vocational school

Some college or graduated from 2-year college with degree

Graduated with degree from 4-year college

Employment status (ref=full time)

Part-time

Unemployed/student/homemaker

Retired

Marital status (ref=not married or in a domestic partnership)

Married or in a domestic partnership

Ever diagnosed by a doctor or health care provider

with high blood pressure or hypertension (ref-yes, diagnosed)

No, had not been ever diagnosed

$1.00(0.86-1.16)$

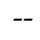

$0.92(0.77-1.10)$

$-$

$1.01(0.79-1.29)$

$--$

$1.01(0.79-1.29)$

$1.02(0.83-1.25)$

$--$

$0.90(0.73-1.12)$

$--$

$1.08(0.81-1.45)$

$-$

$1.11(0.90-1.37)$

$-$

$1.14(0.94-1.37)$

$-$

$1.12(0.90-1.40)$

$-$

$1.03(0.83-1.28)$

$-$

$1.12(0.89-1.40)$

$--$

$1.10(0.95-1.27)$

Self-reported health status (ref-good/fair/poor)

Excellent/very good

$-$

$0.99(0.83-1.17)$

$0.94(0.79-1.11)$

Self-reported weight management status (ref-trying to lose weight)

Not trying to lose weight

Perceived physical activity level (ref=not very active) Active

$-$

$0.77(0.66-0.91)^{* *}$

Calorie and sodium knowledge (ref=low knowledge)

Medium knowledge

High knowledge

Very high knowledge

Number of fast-food and/or sit-down restaurant meals

eaten per week

When ordering food at a restaurant, level of importance of its nutri-

tional content

Frequency of using food label or nutrition facts label when buying

packaged products (ref=never to about half the time)

Always/most of the time

$-$

$-$

$1.05(0.88-1.25)$

$1.27(1.03-1.56)^{*}$

$\begin{array}{ll}-- & 1.02(0.72-1.44) \\ - & 1.00(0.98-1.03)\end{array}$

Currently watching or reducing salt intake (ref=no, not currently

watching or reducing)

Yes, currently watching or reducing

a IRR = Incidence Rate Ratio; 95\% CI = 95\% Confidence Interval.

${ }^{*} \mathrm{p}<0.05^{* *} \mathrm{p}<0.001{ }^{* * *} \mathrm{p}<0.0001$

Table 4. Association between the cumulative sum of adopted modifiable lifestyle behaviors to prevent/manage diabetes and brief lifestyle intervention exposure status: Results from a 2016 internet panel survey of Los Angeles County residents ( $\mathrm{n}=953$ )

\section{Dependent Variable}

$\operatorname{IRR}(95 \% C I)^{a}$

Cumulative sum of adopted modifiable lifestyle behaviors to pre-

vent/manage diabetes

\section{Primary Regressor}

Number of lifestyle behaviors that a doctor or

health care provider recommended to improve on 


\section{Covariates}

Sex (ref=male)

Female

Age (ref $=18-44$ years)

45-64 years

65 years or older

Race/ethnicity (ref=White)

Black

Hispanic/Latino

ANHOPI

Education status (ref=obtained professional degree)

High school or less/vocational school

Some college or graduated from 2-year college with degree

Graduated with degree from 4-year college

Employment status (ref=full time)

Part-time

Unemployed/student/homemaker

Retired

Marital status (ref=not married or in a domestic partnership)

Married or in a domestic partnership

$0.99(0.85-1.15)$

$0.90(0.75-1.08)$

$0.99(0.77-1.27)$

$1.07(0.83-1.37)$

$1.04(0.86-1.26)$

$-$

$0.98(0.79-1.22)$

$1.02(0.77-1.34)$

Ever diagnosed by a doctor or health care provider

with high blood pressure or hypertension (ref-yes, diagnosed)

No, had not been ever diagnosed

$1.18(1.00-1.40)$

Self-reported health status (ref=good/fair/poor) Excellent/very good

Self-reported weight management status (ref-trying to lose weight) Not trying to lose weight

Perceived physical activity level (ref=not very active) Active

Calorie and sodium knowledge (ref=low knowledge)

Medium knowledge

High knowledge

Very high knowledge

Number of fast-food and/or sit-down restaurant meals

eaten per week

When ordering food at a restaurant, level of importance of its nutri-

$1.05(0.89-1.24)$

$1.16(0.95-1.41)$

$0.99(0.71-1.38)$

tional content

$1.04(1.02-1.06)^{* * *}$

Frequency of using food label or nutrition facts label when buying

packaged products (ref=never/less than half the timelabout half the time) Always/most of the time

Currently watching or reducing salt intake (ref=no, not currently watching or reducing)

Yes, currently watching or reducing

a IRR = Incidence Rate Ratio; 95\% CI = 95\% Confidence Interval.

${ }^{*} \mathrm{p}<0.05^{* *} \mathrm{p}<0.001^{* * *} \mathrm{p}<0.0001$ 
Table 5. Association between the cumulative sum of adopted modifiable lifestyle behaviors to prevent/manage diabetes and recent diabetes provider-patient communication: Results from a 2016 internet panel survey of Los Angeles County residents $(\mathrm{n}=953)$

\section{Characteristics}

Dependent Variable

Cumulative sum of adopted modifiable lifestyle behaviors to prevent/manage diabetes

\section{Primary Regressor}

Spoke with a doctor or health care provider about pre-diabetes or diabetes at least once in the last year (ref=no, has not spoken with a health care provider in the past year)

Yes, has spoken with a health care provider in the past year

\section{Covariates}

Sex (ref=male)

Female

Age (ref $=18-44$ years)

45-64 years

65 years or older

Race/ethnicity (ref=White)

Black

Hispanic/Latino

ANHOPI

Education status (ref=obtained professional degree)

High school or less/vocational school

Some college or graduated from 2-year college with degree

Graduated with degree from 4-year college

Employment status (ref=full time)

Part-time

Unemployed/student/homemaker

Retired

Marital status (ref=not married or in a domestic partnership)

Married or in a domestic partnership

Ever diagnosed by a doctor or health care provider

with high blood pressure or hypertension (ref=yes, diagnosed)

No, had not been ever diagnosed

Self-reported health status ( $\mathrm{ref}=$ good/fair/poor)

Excellent/very good

Self-reported weight management status (ref=trying to lose weight)

Not trying to lose weight

Perceived physical activity level (ref=not very active) Active

Calorie and sodium knowledge (ref=low knowledge)

Medium knowledge

High knowledge

Very high knowledge

Number of fast-food and/or sit-down restaurant meals

eaten per week

When ordering food at a restaurant, level of importance of its nutritional content

Frequency of using food label or nutrition facts label when buying

packaged products (ref=never/less than half the time/about half the time)

Always/most of the time

Currently watching or reducing salt intake (ref=no, not currently

watching or reducing)

Yes, currently watching or reducing

a IRR = Incidence Rate Ratio; 95\% CI = 95\% Confidence Interval.

${ }^{*} \mathrm{p}<0.05^{* *} \mathrm{p}<0.001^{* * *} \mathrm{p}<0.0001$
$1.84(1.58-2.14)^{* * *}$

$1.53(1.31-1.79)^{* * *}$

$1.02(0.88-1.19)$

$0.98(0.82-1.17)$

$1.10(0.86-1.41)$

$1.05(0.82-1.34)$

$1.00(0.83-1.21)$

$0.90(0.73-1.12)$

$1.02(0.78-1.33)$

$1.07(0.87-1.32)$

$1.11(0.92-1.34)$

$1.17(0.95-1.44)$

$1.03(0.83-1.27)$

1.12 (0.90-1.38)

$1.07(0.93-1.23)$

1.05 (0.89-1.24)

0.93 (0.79-1.10)

$0.76(0.66-0.88)^{* * *}$

1.04 (0.87-1.26)

$1.07(0.91-1.26)$

$1.30(1.08-1.57)^{* *}$

$1.14(0.79-1.63)$

$1.00(0.98-1.02)$

$1.03(1.01-1.06)^{* *}$

1.09 (0.93-1.28)

$1.42(1.20-1.69)^{* * *}$ 


\section{Discussion}

A myriad of factors can influence individuals' lifestyle behaviors, from intrapersonal characteristics (e.g., individuals' knowledge/attitudes) to broader macrolevel determinants of health (e.g., physical, social, and food environments) [25]. As such, over the last decade, a variety of multi-pronged approaches have been employed to improve adherence to lifestyle recommendations from U.S. health and public health authorities. These have ranged from implementation of nutrition- and physical activityrelated policy, system, and environmental change interventions (PSEs) [26] to scaling of evidence-based lifestyle behavior programs such as the National Diabetes Prevention Program [27]. The latter, however, can be resource and time-intensive, and difficult to establish in the community setting [28]. However, in recent years brief lifestyle interventions such as the "Small Steps for Big Changes Program" have sought to address existing resource and time constraints typically encountered in T2DM lifestyle programs [29].

Finding additional opportunities to better leverage existing infrastructures (e.g., health care settings, etc.) and coupling it to macro-level PSEs - or building upon existing brief lifestyle interventions that seek to reduce T2DM through brief lifestyle counseling sessions - will be essential for lowering the risk of T2DM and its costly complications. This combination-driven approach is not necessarily new to health services or public health, but it does require re-imagining of how clinical care and population health practice fit together, recognizing that a combination of lifestyle changes alongside medical care tailored to the whole person and PSEs targeted at socioeconomic drivers of health inequities are all needed to optimize services for individuals with prediabetes and/or diabetes. Recent U.S. Prevention Services Task Force recommendations support this rationale [30]; they also highlight the importance of provider-patient interactions/communication alongside its previous recommendations on nutrition and physical activity [31] and intervening on social needs [32]. The pursuit of these lifestyle change strategies have global implication beyond what is taking place in the U.S. [33-35].

Within this context, the present study sought to better understand how different types of provider-patient interactions can influence individuals' adoption of lifestyle behaviors to prevent and/or manage T2DM, recognizing that when these interactions are used strategically to encourage individuals to act, the risk of diabetes and its complications become substantively more preventable and manageable. Two notable study findings speak to this observation.

First, prevention approaches in the form of provider encouragement to individuals to improve and expand their lifestyle behavior choices cumulatively appear feasible and may offer individuals several meaningful pathways to better T2DM control. That is, in the present study, all three provider-patient interactions examined (i.e., lifetime prediabetes/diabetes diagnosis status, brief lifestyle intervention exposure, and recent diabetes provider-patient communication) were associated with a higher cumulative sum of adopted modifiable lifestyle behaviors (behavioral changes) among the survey respondents. In addition, results from the multivariable regression models suggest that all three interactions were not necessarily equal in their magnitude nor contribution to the overall impact (odds ratios varied), indicating that the combinations of these interactions may have additive effects; further research will be needed to further explore this. Overall, this survey finding points to the possibility of using these provider-patient interactions to further optimize the care of prediabetic/diabetic patients through an emphasis or focus on making several lifestyle changes simultaneously. Previous studies on brief intervention by physicians in the areas of smoking cessation and problem drinking further lend support for this type of approach $[6,7,11]$. 
Second, study results showed that nutrition knowledge, attitudes, and prior health behavior patterns can be critical for helping individuals with diabetes prevention and management. Considering the nutritional content of food when making restaurant purchasing decisions, for example, was associated with a higher cumulative sum of adopted modifiable lifestyle behaviors (behavioral changes) in the survey. These results provide insights into and are consistent with a growing body of literature that indicates nutrition knowledge, self-efficacy, and quality of food environments all play important roles in shaping how and what people eat [36-39]. In this regard, investing in individual (microlevel) approaches to combat T2DM remains a critical action. These individual-level interventions should complement, instead of compete with, the sometimes more popular policy/system (macro-level) interventions that have been implemented by federal and state public health agencies during the past two decades, including those focused on childhood obesity prevention [40-42] and other health promotion in the general population [43-45].

\section{Limitations}

The overall design and output of the internet panel survey were subject to several limitations. First, recall bias and social desirability bias may have been introduced into the survey by the very nature of the internet panel development process. For example, compared to the general population, enrolled respondents were probably more comfortable with online surveys. Second, due to the web-based platform, there may have been imbalances in the overall demographic composition of the sample. Namely, respondents who replied to the online survey were more likely to be from a higher socioeconomic status (SES) and had better access to the internet. Although, efforts were made during the study to reduce this possibility, using sociodemographic quotas in the recruitment process to ensure that some of the lower SES groups were given a chance to participate. Third, some of the survey questions were internally developed, potentially reducing the reliability and validity of these items. However, pretesting was carried out before field implementation, which should have help mitigated this concern. Fourth, the assessment of respondent nutrition knowledge did not capture the full breadth of potential measures of knowledge (e.g., knowledge of respondents' ability to identify various types of high sodium foods). Finally, as with most web-based and population-based surveys, calculating a true response rate was challenging; as such, a participation rate was estimated instead. The final participation rate of the internet panel survey was comparable to those of other cross-sectional surveys previously conducted in Los Angeles County $[46,47]$.

\section{Conclusions}

The global burden of T2DM is indisputable. A recent analysis of epidemiologic data from the 2017 Global Burden of Disease Study found that over 6\% of the global population is afflicted by this disease, making it the ninth leading cause of morbidity and mortality in the world [5]. Data from this same study also forecasted that the global prevalence of T2DM will continue to climb, making it a growing public health threat for years to come [5]. To respond to this, the health care system and public health infrastructure in the U.S. and around the world will need to improve on the quality of its efforts to design and implement better, more mutually-reinforcing combinations of micro-and macrolevel interventions. The benefits of provider advice and other provider-patient interactions in the health care setting, for example, could be coupled to PSEs that target socioeconomic drivers of inequities among patients, making clinical care and addressing social 
needs a linked endeavor rather than as separate interventions delivered by different sectors of providers. The urgent need to innovate and improve diabetes prevention and control is no more evident than during the most recent COVID-19 pandemic where uncontrolled T2DM represents a major risk factor for severe SARS-CoV-2 infection and its consequent pathway to hospitalization and death in the U.S. [48, 49] and globally [50, 51].

Author Contributions: B.R. and T.K. conceptualized the initial survey design. B.R. was responsible for data collection, analyses, and writing of the original draft of the manuscript. T.K. provided iterative feedback and helped to review and edit the manuscript for intellectual content. All authors contributed to the interpretation and presentation of the data and helped to finalize the article for publication. All authors have read and approved this final version of the article.

Funding: This project was supported in part by a cooperative agreement from the Centers for Disease Control and Prevention (CDC, U58DP004927).

Institutional Review Board Statement: The project was reviewed and approved by the Los Angeles County Department of Public Health Institutional Review Board.

Informed Consent Statement: The internet panel survey utilized implied consent when eligible panel subscribers clicked on the survey link and completed the online questionnaire.

Data Availability Statement: Available from the authors if data request is appropriate and feasible.

Acknowledgments: The authors thank Global Strategy Group and its sampling partner, Research Now, for their technical support of respondent recruitment and data collection for the internet panel survey. The decisions to analyze and publish the results were those of the authors and were not influenced by the funder nor by any of the affiliated institutions. The contents presented do not necessarily represent the views or positions of the CDC, the University of California, Los Angeles, the local health department, or any other organizations mentioned in the text.

Conflicts of Interest: The authors declare no conflict of interest.

\section{References}

1. Zheng, Y.; Ley, S.H.; Hu, F.B. Global aetiology and epidemiology of type 2 diabetes mellitus and its complications. Nat Rev Endocrinol 2018, 14(2), 88-98.

2. Rippe, J.M. Lifestyle strategies for risk factor reduction, prevention, and treatment of cardiovascular disease. Am J Lifestyle Med 2018, 13(2), 204-212.

3. Benjamin E.J.; Muntner, P.; Alonso, A.; Bittencourt, M.S.; Callaway, C.W.; Carson, A.P.; et al. Heart disease and stroke statistics2019 Update: A report from the American Heart Association. Circulation 2019, 5, 139(10), e56-e528.

4. Angell, S.Y.; McConnell, M.V.; Anderson, C.A.M.; Bibbins-Domingo, K.; Boyle, D.S.; Capewel, S.; et al. The American Heart Association 2030 Impact Goal: A presidential advisory from the American Heart Association. Circulation 2020, 141(9), e120-e138.

5. Khan, M.A.B.; Hashim, M.J.; King, J.K.; Govender, R.D.; Mustafa, H.A.; Kaabi, J. Epidemiology of type 2 diabetes - global burden of disease and forecasted trends. J Epidemiol Glob Health 2020, 10(1), 107-111.

6. Fleming, M.F.; Mundt, M.P.; French, M.T.; Manwell, L.B.; Stauffacher, E.A.; Barry, K.L. Brief physician advice for problem drinkers: long-term efficacy and benefit-cost analysis. Alcohol Clin Exp Res 2002, 26(1), 36-43.

7. Engler, P.A.; Ramsey, S.E.; Smith, R.J. Alcohol use of diabetes patients: the need for assessment and intervention. Acta Diabetol 2013, 50(2), 93-9.

8. Xiang, X.; Hernandez, R.; Larrison, C.R. Provider advice on exercise and diet among adults with comorbid serious psychological distress and diabetes or diabetes risk factors. Diabetes Educ 2015, 41, 185-193.

9. Anokye, N.K.; Lord, J.; Fox-Rushby, J. Is brief advice in primary care a cost-effective way to promote physical activity? Br J Sports Med 2014, 48(3), 202-6.

10. Rose, S.A.; Poynter, P.S.; Anderson, J.W.; Noar, S.M.; Conigliaro, J. Physician weight loss advice and patient weight loss behavior change: a literature review and meta-analysis of survey data. Int J Obes (Lond) 2013, 37(1), 118-28.

11. Fleming, M.F.; Balousek, S.L.; Grossberg, P.M.; Mundt, M.P.; Brown, D.; Wiegel, J.R.; Zakletskaia, L.I.; Saewyc, E.M. Brief physician advice for heavy drinking college students: a randomized controlled trial in college health clinics. J Stud Alcohol Drugs 2010, 71(1), 23-31.

12. Bardach, S.H.; Schoenberg, N.E. The role of primary care providers in encouraging older patients to change their lifestyle behaviors. Clin Gerontol 2018, 41(4), 326-334. 
13. Garfield, S.A.; Malozowski, S.; Chin, M.H.; Narayan, K.M.; Glasgow, R.E.; Green, L.W.; et al. Considerations for diabetes translational research in real-world settings. Diabetes Care 2003, 26(9), 2670-4.

14. Aziz, Z.; Absetz, P.; Oldroyd, J.; Pronk, N.P.; Oldenburg, B. A systematic review of real-world diabetes prevention programs: learnings from the last 15 years. Implement Sci 2015, 15, 10:172.

15. Dewey, G.; Wickramasekaran, R.N.; Kuo, T.; Robles, B. Does sodium knowledge affect dietary choices and health behaviors? Results from a survey of Los Angeles County residents. Prev Chronic Dis 2017, 14, E120.

16. US Census Bureau. 2013 American Community Survey questionnaire. 2013. Available online at: http://www2.census.gov/programs-surveys/acs/methodology/questionnaires/2013/quest13.pdf. Accessed November 29, 2021)

17. Los Angeles County Department of Public Health. 2011 Los Angeles County Health Survey background summary and methodology. http://www.publichealth.lacounty.gov/ha/LACHSBackMeth2011.htm. Accessed November 29, 2021.

18. Hays, R.D.; Liu, H.; Kapteyn, A. Use of Internet panels to conduct surveys. Behav Res Methods 2015, 47(3), 685-90.

19. Loef, M.; Walach, H. The combined effects of healthy lifestyle behaviors on all cause mortality: a systematic review and metaanalysis. Prev Med 2012, 55(3), 163-70.

20. Ng, R.; Sutradhar, R.; Yao, Z.; Wodchis, W.P.; Rosella, L.C. Smoking, drinking, diet and physical activity-modifiable lifestyle risk factors and their associations with age to first chronic disease. Int J Epidemiol 2020, 49(1), 113-130.

21. Feldman, A.L.; Long, G.H.; Johansson, I.; Weinehall, L.; Fhärm, E.; Wennberg, P.; et al. Change in lifestyle behaviors and diabetes risk: evidence from a population-based cohort study with 10 year follow-up. Int J Behav Nutr Phys Act 2017, 14(1), 39.

22. D'Addario, L.; Kuo, T.; Robles, B. Do knowledge about sodium, health status by self-report, and having hypertension predict sodium consumption behaviors among Southern California hospital employees? Transl Behav Med 2021, 11(6), 1254-1263.

23. Izquierdo, A.; Ong, M.; Pulido, E.; Wells, K.B.; Berkman, M.; Linski, B.; et al. Community partners in care: 6- and 12-month outcomes of community engagement versus technical assistance to implement depression collaborative care among depressed older adults. Ethn Dis 2018, 28(Suppl 2), 339-348.

24. Gase, L.N.; Montes, C.; Robles, B.; Tyree, R.; Kuo, T. Media outlet and consumer reactions to promotional activities of the Choose Health LA Restaurants Program in Los Angeles County. J Public Health Manag Pract 2016, 22(3), 231-44.

25. Golden, S.D.; Earp, J.A. Social ecological approaches to individuals and their contexts: twenty years of health education \& behavior health promotion interventions. Health Educ Behav 2012, 39(3), 364-72.

26. Kumanyika, S.K. A framework for increasing equity impact in obesity prevention. Am J Public Health 2019, 109(10), $1350-1357$.

27. Ritchie, N.D.; Baucom, K.J.W.; Sauder, K.A. Benefits of participating with a partner in the National Diabetes Prevention Program. Diabetes Care 2020, 43(2), e20-e21.

28. DeFosset, A.R.; Sivashanmugam, M.; Mosst, J.T.; Kuo, T. Clinic- and community-based National Diabetes Prevention Programs in Los Angeles. Health Educ Behav 2021, 1-11 (Online ahead of print).

29. The University of British Columbia. Small Steps for Big Changes- About Us (2022). Available online at: https://ok-smallsteps.sites.olt.ubc.ca/about/. Accessed February 8, 2022.

30. US Preventive Services Task Force; Davidson, K.W.; Barry, M.J.; Mangione, C.M.; Cabana, M.; Caughey, A.B.; et al. Screening for prediabetes and type 2 Diabetes: US Preventive Services Task Force Recommendation Statement. JAMA 2021, 326(8), 736743.

31. US Preventive Services Task Force; Krist, A.H.; Davidson, K.W.; Mangione, C.M.; Barry, M.J.; Cabana, M.; et al. Behavioral counseling interventions to promote a healthy diet and physical activity for cardiovascular disease prevention in adults with cardiovascular risk factors: US Preventive Services Task Force Recommendation Statement. JAMA 2020, 324(20), $2069-2075$.

32. Davidson, K.W.; Krist, A.H.; Tseng, C.W.; Simon, M.; Doubeni, C.A.; Kemper, A.R.; et al. Incorporation of social risk in US Preventive Services Task Force recommendations and identification of key challenges for primary care. JAMA 2021, 326(14), 1410-1415.

33. Richardson, E.; Zalatel, J.; Nolte, E. on behalf of Joint Action. National Diabetes Plans in Europe: What lessons are there for the prevention and control of chronic diseases in Europe? 2016. In: Richardson E. (editor) Policy Brief, ISSN 1997-8065. National Institute of Public Health; Copenhagen; World Health Organization, Regional Office for Europe, Ljubljana.

34. Cosentino, F.; Grant, P.J.; Aboyans, V.; Bailey, C.J.; Ceriello, A.; Delgado; et al. 2019 ESC Guidelines on diabetes, pre-diabetes, and cardiovascular diseases developed in collaboration with the EASD: The Task Force for diabetes, pre-diabetes, and cardiovascular diseases of the European Society of Cardiology (ESC) and the European Association for the Study of Diabetes (EASD). Eur Heart J 2020, 41(2), 255-323.

35. Bell, K.; Shaw, J.E.; Maple-Brown, L.; Ferris, W.; Gray, S.; Murfet, G.; Flavel, R.; Maynard, B.; Ryrie, H.; Pritchard, B.; Freeman, R.; Gordon, B.A. A position statement on screening and management of prediabetes in adults in primary care in Australia. Diabetes Res Clin Pract 2020, 164, 108188.

36. Barzegari, A.; Ebrahimi, M.; Azizi, M. A study of nutrition knowledge, attitudes and food habits of college students. J World Appl Sci 2011, 15(7), 1012-1017.

37. Caspi, C.E.; Sorensen, G.; Subramanian, S.V.; Kawachi, I. The local food environment and diet: a systematic review. Health Place 2012, 18(5), 1172-1187. 
38. Bonaccio, M.; Di Castelnuovo, A.; Costanzo, S.; De Lucia, F.; Olivieri, M.; Donati, M.B.; et al. Nutrition knowledge is associated with higher adherence to Mediterranean diet and lower prevalence of obesity. Results from the Moli-sani study. Appetite 2013, 68, 139-46.

39. Muturi, N.; Kidd, T.; Daniels, A.M.; Kattelmann, K.K.; Khan, T.; Lindshield, E.; et al. Examining the role of youth empowerment in preventing adolescence obesity in low-income communities. J Adolesc 2018, 68, 242-251.

40. Anderson, C.E.; Crespi, C.M.; Wang, M.C.; Whaley, S.E.; Chaparro, M.P. The neighborhood food environment modifies the effect of the 2009 WIC food package change on childhood obesity in Los Angeles County, California. BMC Public Health 2020, 20(1), 678.

41. Wang, M.C.; Crespi, K.; Jiang, L.H.; Nobari, T.; Roper-Fingerhut, H.; Rauzon, S.; Robles, B.; Blocklin, M.; Davoudi, M.; Kuo, T.; MacLeod, K.; Seto, E.; Whaley, S.; Prelip, M. Developing an index of dose of exposure to early childhood obesity community interventions. Prev Med 2018, 111, 135-141.

42. Robles, B.; Jiang, L.; Prelip, M.; Nobari, T.Z.; Wang, A.; Kuo, T.; Wang, M.C. Notes from the field: Creating a typology of childhood obesity intervention strategies. Eval Health Prof 2020, 43(2), 131-134.

43. Honeycutt, A.A.; Khavjou, O.A.; Bradley, C.; Neuwahl, S.; Hoerger, T.J.; Bellard, D.; Cash, A.J. Intervention costs from Communities Putting Prevention to Work. Prev Chronic Dis 2016, 13, E98.

44. Kuo, T.; Barragan, N.C.; Readhead, H. Public health investment in team care: Increasing access to clinical preventive services in Los Angeles County. Front Public Health 2018, 6:17.

45. Yarnoff, B.; Bradley, C.; Honeycutt, A.A.; Soler, R.E.; Orenstein, D. Estimating the relative impact of clinical and preventive community-based interventions: An example based on the Community Transformation Grant Program. Prev Chronic Dis 2019, 16, E87.

46. Du, Y.; Shih, M.; Lightstone, A.S.; Baldwin, S. Hypertension among Asians in Los Angeles County: Findings from a multiyear survey. Prev Med Rep 2017, 6, 302-306.

47. Simon, P.A.; Wold, C.M.; Cousineau, M.R.; Fielding, J.E. Meeting the data needs of a local health department: the Los Angeles County Health Survey. Am J Public Health 2001, 91(12), 1950-2.

48. Zhou, Y.; Chi, J.; Lv, W.; Wang, Y. Obesity and diabetes as high-risk factors for severe coronavirus disease 2019 (Covid-19). Diabetes Metab Res Rev 2021, 37(2), e3377.

49. Centers for Disease Control and Prevention. COVID-19. People with certain medical conditions. (2021). Available online at: https://www.cdc.gov/coronavirus/2019-ncov/need-extra-precautions/people-with-medical-conditions.html (accessed November 29, 2021).

50. Shenoy, A.; Ismaily, M.; Bajaj, M. Diabetes and covid-19: a global health challenge. BMJ Open Diabetes Res Care 2020, 8(1), e001450.

51. Bain, S.C.; Czernichow, S.; Bøgelund, M.; Madsen, M.E.; Yssing, C.; McMillan, A.C.; et al. Costs of COVID-19 pandemic associated with diabetes in Europe: a health care cost model. Curr Med Res Opin 2021, 37(1), 27-36. 